\title{
Article
}

\section{The National Project on Achievement in Twins}

\author{
Sara A. Hart ${ }^{1,2}$, Kim Martinez ${ }^{1}$, Patrick C. Kennedy ${ }^{3}$, Colleen M. Ganley ${ }^{1,4}$ and Jeanette Taylor ${ }^{1}$ \\ ${ }^{1}$ Department of Psychology, Florida State University, Tallahassee, FL, USA, ${ }^{2}$ Florida Center for Reading Research, Florida State University, Tallahassee, FL, USA, \\ ${ }^{3}$ Center on Teaching \& Learning, University of Oregon, Eugene, OR, USA and ${ }^{4}$ Learning Systems Institute, Florida State University, Tallahassee, FL, USA
}

\begin{abstract}
The National Project on Achievement in Twins (NatPAT) began in 2017 as part of the third funding cycle of the Florida Learning Disabilities Research Center, a program project grant funded by the Eunice Kennedy Shriver National Institute of Child Health and Development. NatPAT will have a nationally representative sample of elementary school-aged twins in the United States. The overall goal of the project is to uncover salient factors, including genetic and environmental influences, which contribute to the co-development of reading and math performance during the critical developmental period of elementary school. Here we present the specific aims, methods and materials, and future directions of the project.
\end{abstract}

Keywords: Twins; USA; national twin project; reading; math

(Received 31 May 2019; accepted 29 July 2019; First Published online 4 October 2019)

The National Project on Achievement in Twins (NatPAT) is a new twin project focusing on early reading and math development that began recruitment in early 2018. NatPAT was funded as part of the larger Florida Learning Disabilities Research Center, supported by a long-standing program project grant investment by the Eunice Kennedy Shriver National Institute of Child Health and Development. Reading and math problems represent an important public health issue for children in that they are associated with various negative outcomes, including difficulties in school and lower adult socioeconomic status (Geary et al., 2012; Ritchie \& Bates, 2013). Of U.S. fourth-grade students, one-fourth fail to reach even partial mastery of grade-level knowledge in reading, and one-fifth fail to reach partial mastery of grade-level knowledge in math (U.S. Department of Education, 2017), highlighting the prevalence of reading and math difficulties in childhood. However, we lack knowledge concerning the nature of the co-development of reading and math performance. What we do know is that children who struggle in reading often also struggle in math (Landerl \& Moll, 2010), so it is important to identify influences on the development of both reading and math. Therefore, the overall goal of the NatPAT project is to uncover salient factors, including genetic and environmental influences, which contribute to the co-development of reading and math performance during the critical developmental period of elementary school. NatPAT will be a large and geographically diverse twin sample, allowing us to focus on understudied and underserved populations. By using a twin project, we examine multiple methodologies, both behavioral and genetic, to provide complementary evidence on the co-development of reading and math.

Author for correspondence: Sara A. Hart, Email: hart@psy.fsu.edu

Cite this article: Hart SA, Martinez K, Kennedy PC, Ganley CM, and Taylor J. (2019) The National Project on Achievement in Twins. Twin Research and Human Genetics 22: 761-764, https://doi.org/10.1017/thg.2019.86

\section{Specific Aims}

The NatPAT sample will be large and diverse and uniquely situated to address the overall goal of the proposed research through four specific aims. First, using a cohort-sequential design starting in kindergarten, we will examine reading and math performance across elementary school years to model genetic and environmental influences on reading and math (co-)development. Second, we will model the genetic and environmental influences on the co-occurrence of reading and math difficulties, while also testing for sex differences. Third, we will capitalize on publicly available data to characterize the environmental contexts related to the (co-) development of reading and math performance. Finally, we will capture important attitudinal dimensions and examine how they are associated with the (co-)development of reading and math performance.

\section{Materials and Methods}

All recruitment materials and methods have been preregistered at the Open Science Framework (https://osf.io/6fw5c/). It is our intention that articles that come from NatPAT will also be preregistered.

\section{Recruiting Twins}

Recruitment is ongoing and will continue for the length of the 5 -year project (September 2017-June 2022). Recruitment occurs using the UO DIBELS Data System (UO DDS), through our relationship with the University of Oregon. The UO DDS is an online repository that contains student performance data on approximately 1.4 million children who attend schools that use the Dynamic Indicators of Basic Early Literacy Skills (DIBELS) and easyCBM math assessment products (University of Oregon Center on Teaching and Learning, 2019). Data in the UO DDS

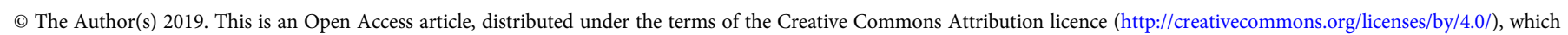
permits unrestricted re-use, distribution, and reproduction in any medium, provided the original work is properly cited. 
Table 1. NatPAT sample characteristics

\begin{tabular}{lccc}
\hline & MZ & DZ & Total \\
\hline Sex & & & \\
\hline Male & 231 & 206 & $437(34.77 \%)$ \\
\hline Female & 191 & 239 & $430(34.21 \%)$ \\
\hline Opposite sex & - & 389 & $389(30.95 \%)$ \\
\hline Not reported & & 1 & $1(0.08 \%)$ \\
\hline Race & & & \\
\hline American Indian or Alaska Native & 1 & 7 & $8(0.64 \%)$ \\
\hline Asian & 4 & 6 & $10(0.80 \%)$ \\
\hline Black or African American & 31 & 65 & $96(7.64 \%)$ \\
\hline Native Hawaiian or Other Pacific Islander & 0 & 2 & $2(0.16 \%)$ \\
\hline White & 355 & 681 & $1036(82.42 \%)$ \\
\hline Mixed/Other & 36 & 69 & $105(8.35 \%)$ \\
\hline Ethnicity & & & \\
\hline Hispanic or Latino & 45 & 84 & $129(10.26 \%)$ \\
\hline Not Hispanic or Latino & 374 & 744 & $1118(88.94 \%)$ \\
\hline Not reported & 3 & 7 & $10(0.80 \%)$ \\
\hline Total & 422 & 835 & 1257 \\
\hline
\end{tabular}

Note: Sample size is twin pairs. $M Z=$ monozygotic; $D Z=$ dizygotic.

include reading and math assessments from kindergarten through sixth grades, with testing typically occurring three times a year.

Starting in January 2018, we began to contact each school in the UO DDS via the phone (the most effective method of those we tried). At contact, we ask school administrative assistants to agree to send home our recruitment materials to any families of multiples in their schools. If the administrative assistant agrees (thus far, approximately $71 \%$ ), we then mail them a pack of our recruitment envelopes (which contain a cover letter explaining the study, a one-page demographic and zygosity questionnaire and a stamped envelope addressed to project staff at Florida State University; these materials are all available on the project page at OSF) along with a $\$ 5$ gift card as a thank-you. All recruitment materials are also available in Spanish, sent upon request.

Families that receive recruitment materials have the option to fill the questionnaire and return it to enroll in the project, and receive a $\$ 10$ gift card for doing so. We then use the demographic information of the twins to pull all available data from the UO DDS, including all previously collected data (and we will update as they age and continue to have new data). Twin zygosity is determined through parent rating of the similarity of the twins using five items. The measure includes two physical characteristics (hair and eye color) and three global similarity ratings (e.g., extent to which relatives could not tell the twins apart; Lykken et al., 1990). This method of recruitment and zygosity determination grew from our previous efforts on the Florida Twin Project on Reading (Taylor \& Schatschneider, 2010) and the Florida Twin Project on Reading, Behavior, and Environment (FTP-RBE; Taylor et al., 2013).

As of this writing, May 2019, we have recruited 1257 twin pairs (see Table 1) who are enrolled in 692 different schools across 43 states (see Figure 1). The twin pairs are 8.64 years old, on average $(S D=2.30$, range $=4.25-14.25)$. At the time of their recruitment, 217 twin pairs were in kindergarten, 215 twin pairs were in first grade, 190 twin pairs were in second grade, 159 twin pairs were in third grade, 183 twin pairs were in fourth grade, 137 twin pairs were in fifth grade, 100 twin pairs were in sixth grade and the remainder were in various grades higher than sixth or pairs were spread between two grades. We will have access to all historical and ongoing data for each twin pair, allowing us to collect longitudinal reading and math data across their elementary school years, for as long as they attend a school that uses the UO DDS.

\section{Reading Measures}

The DIBELS Next is a set of seven benchmark progress monitoring reading measures given in kindergarten through sixth grades, administered individually and designed to be short (Good \& Kaminski, 2011). Not all of the measures are administered in all of the grades; as children become proficient in some more foundational reading skills, some of the measures are designed to be phased out and other measures focused on more complex skills introduced.

First Sound Fluency (FSF). FSF is a measure of phonological awareness that assesses a child's ability to recognize and produce the initial sound in an orally presented word. The examiner says a word to the child, and asks the child to name the beginning sound or group of sounds in that word. The total FSF score is the number of correct beginning sounds produced. This measure is given twice in kindergarten.

Letter Naming Fluency (LNF). LNF is a measure of print awareness that measures a child's ability to rapidly identify upper- and lowercase letter of the alphabet arranged in a random order. The total FSF score is the number of correct letter named correctly. This measure is given three times in kindergarten and once in first grade.

Phoneme Segmentation Fluency (PSF). PSF is a measure of phonological awareness that measures a child's ability to segment words into phonemes. The examiner says a three- or four-phoneme word to the child, and asks the child to say each phoneme individually. The total PSF score is the number of phonemes correctly said. This measure is given twice in kindergarten and one time in first grade.

Nonsense Word Fluency (NWF). NWF is a measure of knowledge of the alphabetic principle - including both letter-sound correspondence and the ability to blend letters into words. Students are presented with a page of nonsense words (e.g., sog) and asked to: (a) say the individual letter sound of each letter, or (b) read the whole nonsense word. For example, if the stimulus word is /sog/ the student could say '/s/ /o/ /g/' to obtain a total of three letter sounds correctly, or say the word '/sog/' to obtain a total of three letter sounds correctly and one whole word read. The final NWF score consists of two parts: (a) the number of letter-sounds produced correctly (CLS) and (b) the number of whole words read (WWR). This measure is given twice in kindergarten, three times in first grade, and once in second grade.

Oral Reading Fluency (ORF). ORF is a measure of decoding and fluency that measures a child's reading accuracy and fluency with connected text. The child reads three passages aloud, and the ORF score is median number of words correctly read across the three passages. An ORF errors score is also created, based on the median number of words omitted, substitutions and hesitations. The ORF score and ORF error score are combined to compute an ORF reading accuracy score. The ORF measure is given twice in first grade and three times per year in first through sixth grades. 


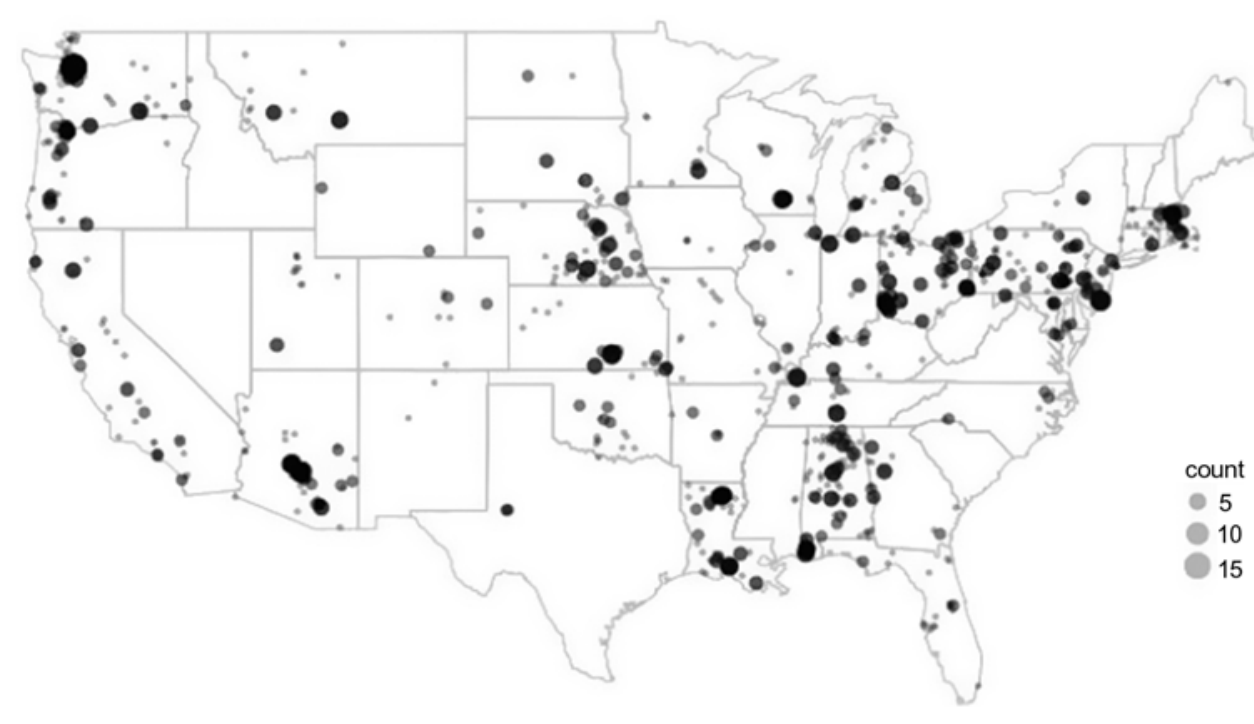

Fig. 1. Map of where participating twin families are located. There are no twin families to date in Alaska or Hawaii.
Retell. Retell is a measure of comprehension, the ability to extract meaning from text. It is assessed using a comprehension question, 'Tell me all that you can about what you just read', after each ORF passage read. The Retell score is composed of the median number of words in the child's response to the retell question that are related to the passage. This measure is given twice in first grade and three times per year in first through sixth grades.

DIBELS-Maze (Daze). Daze is a measure of reading comprehension. Children read a passage that has approximately every seventh word deleted and replaced with a blank. The children read the passage silently, and complete the sentences by circling one of three word choices on the page. The Daze score is the number of correct words selected, corrected for guessing. This measure is given three times per year in third through sixth grades.

\section{Math Measures}

The easyCBM Math measure is grade-level measure of math content knowledge and skills, given three times a year from kindergarten to eighth grades. There are two versions of the easyCBM, easyCBM CCSS Math, based on the Common Core State Standards, and easyCBM NCTM Math, based on the National Council for Teachers of Mathematics Focal Point Standards. The content tested by the easyCBM Math measure changes at each testing point, as it is aligned to what children are learning as part of the scope and sequence that each test is aligned to (CCSS or NCTM). Generally, the test items focus on numbers and operations, geometry, measurement, algebra, data analysis and ratios. The test items are multiple choice, and testing occurs online. This measure is given three times per year in kindergarten through eighth grades.

\section{Contextual Data}

For each twin family, we know their home address and school name and address. With this information, we will be able to capitalize on many rich publicly available databases, such as www.data. gov and www.census.gov, as well as use geographic information system (GIS) technology, to quantify various aspects of the twins' context. These contexts will include home (i.e., family income level), classroom (i.e., teacher demographics, average class gains across a school year), school (i.e., school demographics, school lunch expenditures), neighborhood (e.g., income level, learning centers, crime) and geographic region.

\section{Questionnaire Data Collection}

During the summer of 2020, we will mail a questionnaire packet to the twins' homes. This packet will contain both parent and child surveys (only children who are at least in third grade will receive a survey). The parent survey will include self-report questionnaires about their reading and math attitudes and home math activities, as well as a questionnaire about their twins' math and reading attitudes and behaviors. Two copies of a child survey will be sent, one for each twin, which will include measures of their attitudes and perceptions of their environment. The family will be given a $\$ 30$ gift card for a completed parent survey, and $\$ 5$ gift card for each completed child survey.

\section{Future Directions}

Currently, NatPAT is in the recruitment phase, with all resources going toward finding and enrolling families of multiples, with an increased effort toward increasing the racial diversity of the sample. This will continue throughout the funding years. Our goal is to recruit a total sample over 3000 twin pairs. In summer 2020, questionnaires will be sent home to all the NatPAT families, and efforts will start at accumulating the contextual data. After that, manuscript writing based on the specific aims will begin.

Acknowledgments. This research was supported by the Eunice Kennedy Shriver National Institute of Child Health \& Human Development Grant HD052120. Views expressed herein are those of the authors and have neither been reviewed nor approved by the granting agencies.

\section{References}

Geary, D. C., Hoard, M. K., Nugent, L., \& Bailey, D. H. (2012). Mathematical cognition deficits in children with learning disabilities and persistent low achievement: A five-year prospective study. Journal of Educational Psychology, 104, 206-223.

Good, R. H., \& Kaminski, R. A. (2011). DIBELS next technical manual. Eugene, OR: Dynamic Measurement Group. Retrieved from https://dibels.org/next/ index.php

Landerl, K., \& Moll, K. (2010). Comorbidity of learning disorders: Prevalence and familial transmission. Journal of Child Psychology and Psychiatry, 51, 287-294. 
Lykken, D. T., Bouchard, T. J., McGue, M., \& Tellegen, A. (1990). The Minnesota twin family registry: Some initial findings. Acta Geneticae Medicae et Gemellologiae: Twin Research, 39, 35-70.

Ritchie, S. J., \& Bates, T. C. (2013). Enduring links from childhood mathematics and reading achievement to adult socioeconomic status. Psychological Science, 24, 1301-1308.

Taylor, J. E., Hart, S. A., Mikolajewski, A. J., \& Schatschneider, C. (2013). An update on the Florida State Twin Registry. Twin Research and Human Genetics, 16, 471-475.
Taylor, J., \& Schatschneider, C. (2010). Genetic influence on literacy constructs in kindergarten and first grade: Evidence from a diverse twin sample. Behavior Genetics, 40, 591-602.

University of Oregon Center on Teaching and Learning. (2019). UO DIBELS ${ }^{\oplus}$ data system. Retrieved from https://dibels.uoregon.edu/

U.S. Department of Education. (2017). NAEP mathematics report card. Retrieved from https://www.nationsreportcard.gov/math_2017/nation/ achievement/?grade $=4$ 